\title{
Cardiometabolic outcomes among schizophrenia patients using antipsychotics: the impact of high weight gain risk vs low weight gain risk treatment
}

\author{
Rezaul Khandker ${ }^{1}$, Farid Chekani ${ }^{1 *}$, Brendan Limone ${ }^{2}$ and Ellen Thiel ${ }^{2}$
}

\begin{abstract}
Objective: Evaluate the prevalence of cardiometabolic conditions among schizophrenia patients before and incidence after initiation of high (HWGR) and low weight gain risk (LWGR) antipsychotic (AP) regimens.

Methods: A retrospective observational cohort study was conducted using administrative claims data from the IBM ${ }^{\circledR}$ MarketScan Commercial and Multi-State Medicaid Databases. Patients with $>1$ medical claim with a diagnosis for schizophrenia and newly initiating AP therapy between 1/1/11-6/30/16 were included. Baseline characteristics were assessed in the 12-months before AP initiation; outcomes over 24-months following AP initiation. Patients were characterized by the AP regimen initiated at the index date. Adherence was defined by a medication possession ratio $>0.8$ (medication on hand for $80 \%$ of follow-up). Multivariate modeling identified predictors of index AP weight gain risk profile and post-index dyslipidemia.
\end{abstract}

Results: Two thousand seven hundred forty-eight commercially-insured and 8,748 Medicaid patients met the inclusion criteria. A majority of patients initiated on atypical AP and approximately 30\% were adherent to their index AP regimen. Within both payers, patients indexing on LWGR AP regimens were more likely to have pre-index diagnoses of cardiometabolic conditions including hypertension, dyslipidemia, and diabetes. Significant predictors of post-index dyslipidemia included AP adherence and pre-index diabetes. Within both payers, odds of initiating HWGR AP regimens were higher among patients with evidence of drug abuse.

Conclusions: There is unmet need for reducing cardiometabolic consequences for patients on AP therapy and this analysis provides evidence that cardiometabolic conditions often develop during early stages of AP therapy. However, this does not appear to be related to the weight gain risk profile of the AP regimen.

Keywords: Schizophrenia, Antipsychotic, Adherence, Cardiometabolic, Dyslipidemia

\section{Introduction}

Schizophrenia is one of the twenty top leading causes of disability across the globe [1]. Schizophrenia is a debilitating, chronic mental illness in which patients exhibit

${ }^{*}$ Correspondence: farid.chekani@merck.com

${ }^{1}$ Merck \& Co., Inc., 2000 Galloping Hill Rd, Kenilworth, NJ 07033, USA

Full list of author information is available at the end of the article hallucinations, delusions, along with possible grossly disorganized or catatonic behavior and negative symptoms (i.e. lack of motivation) [2]. Current treatment focuses on reducing symptom burden and preventing relapse [3]. These symptoms, paired with commonly occurring comorbid psychiatric conditions, such as substance abuse and anxiety disorders, lead to social and occupational dysfunction. 
Antipsychotic medications are the primary pharmacologic treatment for schizophrenia. Antipsychotics reduce the severity of psychotic symptoms and the incidence of relapse in most patients; [3] however, other health conditions and excess mortality still pose a significant burden in this population. Patients with schizophrenia die on average 25 years earlier than the general population, and most of the excess mortality is due to premature cardiovascular deaths related to dyslipidemia [4]. Schizophrenia patients tend to make little use of health care resources despite poor physical health, [5] which may lead to under-detection of preexisting cardiometabolic comorbidities and risk factors. Therefore, it is important to continue to study the associations between antipsychotics and cardiovascular health among schisoprehnia patients.

It is well documented that most antipsychotics cause some amount of weight gain [6]. Clozapine and olanzapine were most likely to cause weight gain, followed closely by risperidone and quetiapine, with aripiprazole and ziprasidone being least likely to lead to significant weight gain [6]. Although antipsychotic medications with high weight gain risk (HWGR) have been identified, direct evidence linking weight gain risk to other cardiometabolic outcomes using real-world data is sparse.

To help address this gap in clinical understanding, the primary objective of this analysis was to evaluate the prevalence of cardiometabolic conditions among schizophrenia patients prior to initiating first-line antipsycotics and the incidence of these conditions following initiation of antipsychotic regimens using real-world data. The type of antipsychotics initiated and whether baseline clinical characteristics are associated with higher likelihood of being prescribed higher or lower weight gain risk medications were analyzed. We also examined whether the weight gain risk profile of the index antipsychotic regimen impacts the incidence of new cardiometabolic conditions.

\section{Methods \\ Sample}

This retrospective cohort study used U.S. administrative claims data from the $\mathrm{IBM}^{\circledR}$ MarketScan ${ }^{\circledR}$ Commercial Claims and Encounters Database for the period between January 1, 2011 and December 31, 2016 and the MarketScan Medicaid Multi-State Database for the period between January 1, 2011 and June 30, 2016. The Commercial Database includes the claims of patients and their families who are insured through their employers. The Medicaid Multi-State Database includes claims of millions of patients insured by Medicaid from multiple states. Medicaid coverage differs from state to state, and generally includes healthcare coverage for low-income individuals and individuals with disabilities. This research is not considered human subject research as it does not involve interactions with - or interventions among participants, and only de-identified data was available. All data were de-identified and fully compliant with the Health Insurance Portability and Accountability Act (HIPAA) of 1996, and therefore the study did not require approval or waiver from an institutional review board [7].

\section{Patient selection criteria}

All patients included were adults initiating antipsychotic therapy for schizophrenia. The schizophrenia diagnosis was defined by one or more inpatient or two or more non-diagnostic (i.e. non rule-out) outpatient claims with a diagnosis of schizophrenia based on International Classification of Diseases (ICD) codes (ICD9-CM 295.0x-295.6x, 295.8x-295.9x; ICD-10-CM F20. $\mathrm{xx})$. Patients were required to have a prescription filled for an antipsychotic medication 6 months prior to, on, or after the first schizophrenia diagnosis. The date of the first pharmacy claim for antipsychotic medication(s) was deemed the index date. Patients were required to be continuously enrolled with medical and prescription drug benefits throughout the 12 months preceding and 24 months following the index date but were not required to meet any minimum number of inpatient or outpatient services throughout the study period.

Patients with claims for any antipsychotic in the 12 months prior to the index date were excluded. This criterion was applied to select patients that were newly initiating AP therapy after at least one year without such therapy. Patients were excluded if they had a claim with a diagnosis for mild cognitive impairment, dementia, or Alzheimer's disease during baseline, or bipolar disorder, psychosis due to medication condition, personality disorder(s) or pervasive developmental disorder(s) during the follow-up period. Patients with pharmacy claims for clozapine were also excluded, since clozapine is indicated for treatment resistant schizophrenia [8].

Patients were categorized by antipsychotics with HWGR versus those with low weight gain risk (LWGR). Patients were classed as being on a HWGR index treatment regimen if they had one or more claims for olanzapine, chlorpromazine, iloperidone, paliperidone, quetiapine, risperidone, or mesoridazine. Conversely, patients were classified as initiating a LWGR index treatment regimen if they had one or more claims for fluphenazine, haloperidol, perphenazine, thioridazine, thiothixene, aripiprazole, asenapine, lurasidone, ziprasidone, brexpiprazole, trifluoperazine, or cariprazine $[6$, 9-11] . Patients with claims for both high and low-risk antipsychotic on the index date were classified in the HWGR cohort. 


\section{Demographic and baseline psychiatric characteristics}

In both payer cohorts, demographic characteristics were measured as of the index date. Baseline characteristics were measured during the 12-month baseline period and were identified by the presence of at least one medical claim with a diagnosis code for the condition. Conditions of interest included schizophrenia subtype diagnosis, anxiety and mood disorders, and substance abuse disorders (drug abuse, alcohol abuse, other substance abuse/ dependency disorder).

\section{Antipsychotic medications and weight gain risk profiles} Patients were characterized in terms of the first-line antipsychotic initiated at the index date. Index treatment adherence was measured during the 24-month follow-up period using the Medication Possession Ratio (MPR). The MPR was defined as the sum of the days supplied of the index antipsychotic divided by the total number of days in the follow-up period; that is, number of days' supply that a patient should have received had they obtained the medication as prescribed. For patients who received two or more APs (i.e., combination therapy), the MPR was calculated by averaging the days' supply of both medications and dividing by the total number of days in the follow-up period. For long-acting injectable (LAI) medications, the days of clinical benefit derived from dosing schedules, was used in place of days supply. MPR values range from 0 to 1 , where a value of 1 corresponds to $100 \%$ adherence. We defined adherence as MPR values equal to or greater than $80 \%$, indicating that patients showed continuous medication use for at least $80 \%$ of days in the 24-month follow-up period.

\section{Cardiometabolic conditions}

Cardiometabolic characteristics were measured during the 12-month baseline period and the 24-month post-index period were identified by the presence of at least one claim with a diagnosis code for the condition. Additionally, we recorded cardiometabolic-related medications received during the baseline and 24-month follow-up period using National Drug Codes; these medications included antihypertensives, antidiabetic medications and lipid lowering agents.

\section{Statistical analysis}

All analyses were conducted separately by payer group (commercially-insured versus Medicaid) and stratified by index antipsychotic regimen weight gain risk profile (HWGR versus LWGR). Separate analyses were conducted from the onset of the study based on the two insurance groups (i.e., Medicaid and Commercial claims) due to: potential differences in antipsychotic prescribing patterns (e.g., types of medication prescribed and days supply), differences in available demographic characteristics (e.g., race only available in Medicaid, region only available in commercial), and differences in the patient cost-sharing for medications which may lead to different adherence metrics. No differences were expected initially in terms of medication adherence and HWGR association across the two insurance groups. Differences between index antipsychotic regimen weight gain risk profile strata (HWGR vs LWGR) were assessed using chi square tests for categorical variables and t-tests or analysis of variance (ANOVA) for continuous variables.

A logistic regression model was fit to identify predictors of a dyslipidemia diagnosis in the follow-up period. The model was fit among the subset of patients who did not have baseline dyslipidemia. A second logistic regression model was fit to identify predictors of a patient initiating HWGR versus LWGR antipsychotics. For all analyses, p-values less than 0.05 were considered statistically significant.

\section{Results}

\section{Descriptive statistics}

\section{Demographic and psychiatric characteristics}

A total of 63,467 commercially-insured schizophrenia patients were identified, of whom 2,748 patients were new initiators of antipsychotics and met the additional eligibility criteria. In the Medicaid database, 218,961 schizophrenia patients were identified, of whom 8,748 met the eligibility criteria (Supplemental Figure 1). The mean (SD) age was 38.7 (18.2) years among commercially-insured patients and 39.9 (13.8) years among Medicaid patients (Table 1). Around 70\% of patients in both payer groups were categorized as initiating a HWGR regimen. Patients prescribed a HWGR regimen were significantly younger and more likely to be male compared with their LWGR counterparts. The most common psychiatric comorbidities included major depressive disorder, drug abuse, and other substance abuse/dependency disorder.

\section{Index antipsychotic medication types, treatment patterns, and adherence}

In both payer groups, over $80 \%$ of patients were prescribed atypical antipsychotics at baseline. The atypical antipsychotic prescribed the most was risperidone. Around 1 in 5 patients were prescribed a combination of antipsychotics. The mean (SD) number of days supply of the index antipsychotic was 35.1 (23.2) in the commercial sample and 27.4 (8.8) in Medicaid. Less than one-third of both the commercially-insured and Medicaid patients ( $29 \%$ and $31.6 \%$, respectively) were defined as adherent to the index regimen.In the Medicaid sample, a higher 
Table 1 Demographic and clinical characteristics at baseline by payer group and index antipsychotic weight gain risk profile

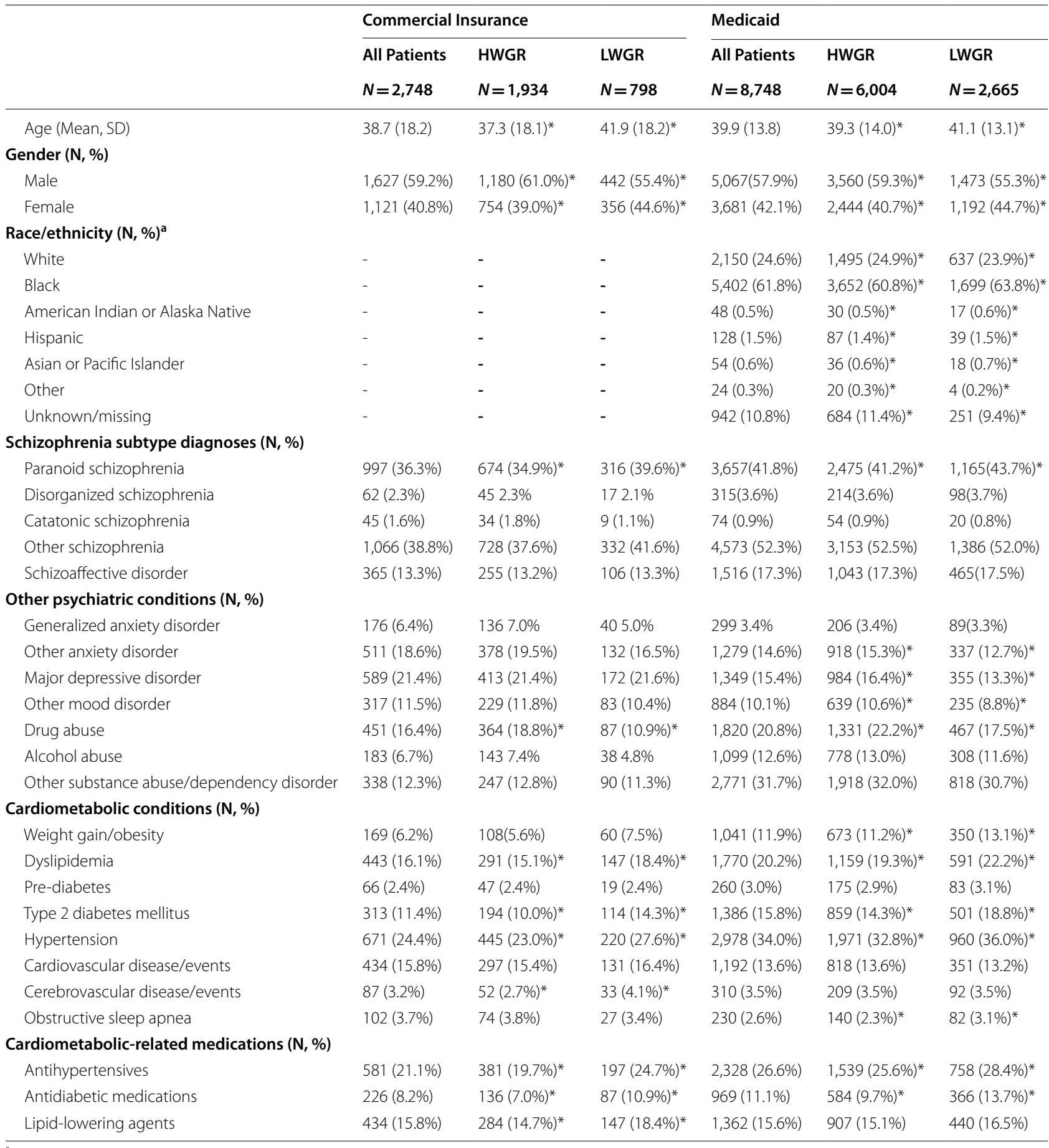

${ }^{*} p<0.05$ between HWGR vs LWGR subgroups

${ }^{a}$ Race/ethnicity data available for Medicaid sample only

Abbreviations: HWGR High weight gain risk index treatment regimen, LWGR Low weight gain risk index treatment regimen, $N$ number, SD Standard deviation

proportion of patients initiating a HWGR regimen were adherent compared to those prescribed a LWGR regimen (33.6\% vs $27.8, p<0.05)$. No such differences in adherence were observed in the commercial population (Table 2).

\section{Pre-index cardiometabolic comorbidities} and cardiometabolic-related medications

The most common baseline cardiometabolic comorbidities were dyslipidemia and hypertension. In both payer 
Table 2 Antipsychotic medication type, supply, and adherence by payer group and index antipsychotic weight gain risk profile

\begin{tabular}{|c|c|c|c|c|c|c|}
\hline & \multicolumn{3}{|c|}{ Commercial Insurance } & \multicolumn{3}{|l|}{ Medicaid } \\
\hline & All Patients & HWGR & LWGR & All Patients & HWGR & LWGR \\
\hline & $N=2,748$ & $N=1,934$ & $N=798$ & $N=8,748$ & $N=6,004$ & $N=2,665$ \\
\hline \multicolumn{7}{|l|}{ Medication type $(\mathrm{N}, \%)$} \\
\hline Second-generation antipsychotics & $2,413(87.8 \%)$ & $1,919(99.2 \%)^{*}$ & $494(61.9 \%)^{*}$ & $7,367(84.2 \%)$ & $5,964(99.3 \%)^{*}$ & $1,393(52.3 \%)^{*}$ \\
\hline Lurasidone & $47(1.7 \%)$ & $16(0.8 \%)^{*}$ & $31(3.9 \%)^{*}$ & $176(2.0 \%)$ & $41(0.7 \%)^{*}$ & $135(5.1 \%)^{*}$ \\
\hline Aripiprazole & $485(17.7 \%)$ & $140(7.2 \%)^{*}$ & $345(43.2 \%)^{*}$ & $924(10.6 \%)$ & $192(3.2 \%)^{*}$ & $732(27.5 \%)^{*}$ \\
\hline Olanzapine & $553(20.1 \%)$ & $553(28.6 \%)^{*}$ & $0(0.0 \%)^{*}$ & $1,209(13.8 \%)$ & $1,209(20.1 \%)^{*}$ & $0(0.0 \%)^{*}$ \\
\hline Paliperidone & $127(4.6 \%)$ & $127(6.6 \%)^{*}$ & $0(0.0 \%)^{*}$ & $791(9.0 \%)$ & $791(13.2 \%)^{*}$ & $0(0.0 \%)^{*}$ \\
\hline Quetiapine & $429(15.6 \%)$ & $429(22.2 \%)^{*}$ & $0(0.0 \%)^{*}$ & $1,532(17.5 \%)$ & $1,532(25.5 \%)^{*}$ & $0(0.0 \%)^{*}$ \\
\hline Risperidone & $1,001(36.4 \%)$ & $1,001(51.8 \%)^{*}$ & $0(0.0 \%)^{*}$ & $2,871(32.8 \%)$ & $2,871(47.8 \%)^{*}$ & $0(0.0 \%)^{*}$ \\
\hline Ziprasidone & $176(6.4 \%)$ & $59(3.1 \%)^{*}$ & $117(14.7 \%)^{*}$ & $669(7.7 \%)$ & $162(2.7 \%)^{*}$ & $507(19.0 \%)^{*}$ \\
\hline Other & $39(1.4 \%)$ & $22(1.1 \%)^{*}$ & $17(2.1 \%)^{*}$ & $138(1.6 \%)$ & $77(1.3 \%)^{*}$ & $61(2.3 \%)^{*}$ \\
\hline First-generation antipsychotics & $521(19.0 \%)$ & $174(9.0 \%)^{*}$ & $331(41.5 \%)^{*}$ & $2,142(24.5 \%)$ & $652(10.9 \%)^{*}$ & $1,421(53.3 \%)^{*}$ \\
\hline Haloperidol & $272(9.9 \%)$ & $100(5.2 \%)^{*}$ & $172(21.6 \%)^{*}$ & $1,451(16.6 \%)$ & $433(7.2 \%)^{*}$ & $1,018(38.2 \%)^{*}$ \\
\hline Thiothixene & $36(1.3 \%)$ & $6(0.3 \%)^{*}$ & $30(3.8 \%)^{*}$ & - & - & - \\
\hline Fluphenazine & $94(3.4 \%)$ & $24(1.2 \%)^{*}$ & $70(8.8 \%)^{*}$ & $370(4.2 \%)$ & $97(1.6 \%)^{*}$ & $273(10.2 \%)^{*}$ \\
\hline Trifluoperazine & $29(1.1 \%)$ & $5(0.3 \%)^{*}$ & $24(3.0 \%)^{*}$ & - & - & - \\
\hline Perphenazine & $45(1.6 \%)$ & $16(0.8 \%)^{*}$ & $29(3.6 \%)^{*}$ & $109(1.3 \%)$ & $29(0.5 \%)^{*}$ & $80(3.0 \%)^{*}$ \\
\hline Chlorpromazine & $26(1.0 \%)$ & $26(1.3 \%)^{*}$ & $0(0.0 \%)^{*}$ & - & - & - \\
\hline Other & $26(1.0 \%)$ & $5(0.3 \%)^{*}$ & $12(1.5 \%)^{*}$ & $199(2.3 \%)$ & $111(1.9 \%)^{*}$ & $73(2.7 \%)^{*}$ \\
\hline \multicolumn{7}{|l|}{ Route of administration $(\mathrm{N}, \%)$} \\
\hline Oral & $2,605(94.8 \%)$ & $1,890(97.7 \%)^{*}$ & $705(88.4 \%)^{*}$ & $7,615(87.1 \%)$ & $5,548(92.4 \%)^{*}$ & $2,042(76.6 \%)^{*}$ \\
\hline LAl & $235(8.6 \%)$ & $143(7.4 \%)^{*}$ & $92(11.5 \%)^{*}$ & $1,694(19.4 \%)$ & $1,006(16.8 \%)^{*}$ & $688(25.8 \%)^{*}$ \\
\hline Other & $76(2.8 \%)$ & $40(2.1 \%)^{*}$ & $30(3.8 \%)^{*}$ & $330(3.8 \%)$ & $95(1.6 \%)^{*}$ & $181(6.8 \%)^{*}$ \\
\hline \multicolumn{7}{|l|}{ Medication supply and regimen type } \\
\hline Index Antipsychotic Days Supply (Mean, SD) & $35.1(23.2)$ & $33.7(21.7)^{*}$ & $38.5(25.7)^{*}$ & $27.4(8.8)$ & $27.9(7.8)^{*}$ & $26.8(10.0)^{*}$ \\
\hline Monotherapy & 2,192 (79.8\%) & $1,427(73.8 \%)^{*}$ & $749(93.9 \%)^{*}$ & $7,158(81.8 \%)$ & $4,624(77.0 \%)^{*}$ & $2,455(92.1 \%)^{*}$ \\
\hline Combination therapy & $556(20.2 \%)$ & $507(26.2 \%)^{*}$ & $49(6.1 \%)^{*}$ & $1,590(18.2 \%)$ & $1,380(23.0 \%)^{*}$ & $210(7.9 \%)^{*}$ \\
\hline \multicolumn{7}{|l|}{ Medication adherence } \\
\hline Adherence $(N, \%)^{\mathrm{a}}$ & $799(29.1 \%)$ & $566(29.3 \%)$ & $230(28.8 \%)$ & $2,763(31.6 \%)$ & $2,016(33.6 \%)^{*}$ & $740(27.8 \%)^{*}$ \\
\hline MPR (Mean, SD) & $0.46(0.37)$ & $0.47(0.37)$ & $046(0.37)$ & $0.49(0.37)$ & $0.51(0.37)^{*}$ & $0.46(0.36)^{*}$ \\
\hline
\end{tabular}

${ }^{*} p<0.05$ between HWGR vs LWGR subgroups

a Patients with an MPR $>0.80$, calculated as the ratio of the number of days' supply of index treatment to the total number of days in the 24-month post-index treatment date

Abbreviations: HWGR High weight gain risk index treatment regimen, LA/ Long-acting injectable, LWGR Low weight gain risk index treatment regimen, MPR Medication possession ratio, $N$ Number, SD Standard deviation

groups, patients who were prescribed a LWGR regimen were significantly more likely to have underlying type 2 diabetes mellitus compared to patients with a HWGR regimen (Commercial: $10.0 \%$ vs $14.3 \%, p<0.05$; Medicaid: $14.3 \%$ vs $18.8 \%, p<0.05)$. In the Medicaid sample only, patients with a LWGR regimen were also significantly more likely to have diagnosed weight gain/obesity $(11.2 \%$ vs $13.1 \%, p<0.05)$, dyslipidemia $(19.3 \%$ vs $22.2 \%, p<0.05)$, and hypertension $(32.8 \%$ vs $36.0 \%, p<0.05)$ compared to their HWGR counterparts (Table 1).

\section{Post-index cardiometabolic comorbidities}

Rates of newly incident dyslipidemia were $15.7 \%$ and $18.0 \%$ in the commercially-insured and Medicaid samples, respectively (Table 3). Similarly, cardiovascular disease or events were diagnosed in $15.8 \%$ and $19.5 \%$ of the commercially-insured and Medicaid cohorts, respectively. Compared with their HWGR counterparts, LWGR patients in the commercially-insured sample were significantly more likely to have a new diagnosis of weight gain/ obesity (9.2\% vs $11.9 \%)$, type 2 diabetes ( $4.0 \%$ vs $6.7 \%)$, or obstructive sleep apnea (2.0 vs $4.8 \%$ ). Among Medicaid 
Table 3 Post-index cardiometabolic comorbidities by payer group and index antipsychotic weight gain risk profile

\begin{tabular}{|c|c|c|c|c|c|c|}
\hline & \multicolumn{3}{|c|}{ Commercial Insurance } & \multicolumn{3}{|l|}{ Medicaid } \\
\hline & All Patients & HWGR & LWGR & All Patients & HWGR & LWGR \\
\hline \multicolumn{7}{|l|}{ Cardiometabolic conditions ${ }^{\mathrm{a}}, \mathrm{N}(\%)$} \\
\hline \multirow[t]{2}{*}{ Weight gain/obesity } & $N=2,579$ & $N=1,826$ & $N=738$ & $N=7,707$ & $N=5,331$ & $N=2,315$ \\
\hline & $259(10.0 \%)$ & $168(9.2 \%)^{*}$ & $88(11.9 \%)^{*}$ & $1,078(14.0 \%)$ & $693(13.0 \%)^{*}$ & $371(16.0 \%)^{*}$ \\
\hline \multirow[t]{2}{*}{ Dyslipidemia } & $N=2,305$ & $N=1,643$ & $N=651$ & $N=6,978$ & $N=4,845$ & $N=2,074$ \\
\hline & $363(15.7 \%)$ & $251(15.3 \%)$ & $109(16.7 \%)$ & $1,253(18.0 \%)$ & $842(17.4 \%)$ & $398(19.2 \%)$ \\
\hline \multirow[t]{2}{*}{ Pre-diabetes } & $N=2,682$ & $N=1,887$ & $N=779$ & $N=8,488$ & $N=5,829$ & $N=2,582$ \\
\hline & $131(4.9 \%)$ & $89(4.7 \%)$ & $42(5.4 \%)$ & $464(5.5 \%)$ & $319(5.5 \%)$ & $138(5.3 \%)$ \\
\hline \multirow[t]{2}{*}{ Type 2 diabetes mellitus } & $N=2,435$ & $N=1,740$ & $N=684$ & $N=7,362$ & $N=5,145$ & $N=2,164$ \\
\hline & $116(4.8 \%)$ & $70(4.0 \%)^{*}$ & $46(6.7 \%)^{*}$ & $628(8.5 \%)$ & $423(8.2 \%)$ & $200(9.2 \%)$ \\
\hline \multirow[t]{2}{*}{ Hypertension } & $N=2,077$ & $N=1,489$ & $N=578$ & $N=5,770$ & $N=4,033$ & $N=1,705$ \\
\hline & $300(14.4 \%)$ & $210(14.1 \%)$ & 89 (15.4\%) & $1,266(21.9 \%)$ & $850(21.1 \%)$ & $404(23.7 \%)$ \\
\hline \multirow[t]{2}{*}{ Cardiovascular disease/events } & $N=2,314$ & $N=1,637$ & $N=667$ & $N=7,556$ & $N=5,186$ & $N=2,314$ \\
\hline & $365(15.8 \%)$ & $249(15.2 \%)$ & $115(17.2 \%)$ & $1,477(19.5 \%)$ & $1,012(19.5 \%)$ & $443(19.1 \%)$ \\
\hline \multirow[t]{2}{*}{ Cerebrovascular disease/events } & $N=2,661$ & $N=1,882$ & $N=765$ & $N=8,438$ & $N=5,795$ & $N=2,573$ \\
\hline & $82(3.1 \%)$ & $54(2.9 \%)$ & $27(3.5 \%)$ & $324(3.8 \%)$ & $210(3.6 \%)$ & 109 (4.2\%) \\
\hline \multirow[t]{2}{*}{ Obstructive sleep apnea } & $N=2,646$ & $N=1,860$ & $N=771$ & $N=8,518$ & $N=5,864$ & $N=2,583$ \\
\hline & $76(2.9 \%)$ & $38(2.0 \%)^{*}$ & $37(4.8 \%)^{*}$ & $254(3.0 \%)$ & $157(2.7 \%)$ & $89(3.4 \%)$ \\
\hline
\end{tabular}

* $p<0.05$ between HWGR vs LWGR subgroups

${ }^{\text {a }}$ Conditions developed during the 24-months following index antipsychotic therapy (Denominators depict total number of patients with no evidence of specified diagnosis during 12-months prior to index date)

Abbreviations: HWGR High weight gain risk index treatment regimen, LWGR Low weight gain risk index treatment regimen, $N$ number

patients, LWGR patients were significantly more likely to have a new diagnosis of weight gain/obesity $(13.0 \%$ vs $16.0 \%)$.

\section{Multivariate analyses \\ Post-index dyslipidemia}

In both payer groups, medication adherence and type 2 diabetes mellitus at baseline were the strongest predictors of dyslipidemia during the follow-up. In the commercially-insured sample, the odds of dyslipidemia were 1.75 (95\% confidence intervals, CI: 1.24-2.47) times higher among patients who adhered to their index antipsychotic regimen and 1.83 (95\% CI: 1.25-2.67) times higher among patients with type 2 diabetes mellitus compared with their counterparts. In the Medicaid sample, the corresponding odds ratios were 2.58 (95\% CI: 2.133.12) for medication adherence and 2.24 (95\% CI: $1.85-$ 2.72) for type 2 diabetes mellitus. Baseline cardiovascular disease/events was also a significant predictor (Table 4).

\section{Predictors of high weight gain risk vs low weight gain risk regimen}

In the Commercial population, the odds of being prescribed a HWGR antipsychotic regimen at index were 1.43 times higher in patients with evidence of drug abuse (95\% CI: $1.07-1.91$ ) and 1.71 times higher in patients with delusional disorders (95\% CI: 1.25-2.34). In the Medicaid population, the odds of being prescribed a HWGR antipsychotic regimen at index were 1.21 times higher among patients with evidence of drug abuse (95\% CI:1.06-1.38) and 1.22 times higher among patients with major depressive disorder (95\% CI: 1.06-1.41) (Table 4).

\section{Discussion}

We used real-world administrative claims data to examine the prevalence and incidence of cardiometabolic conditions among schizophrenia patients prior to and following initiation of their antipsychotic regimen. We found high rates of newly incident dyslipidemia, hypertension, and cardiovascular events regardless of payer, suggesting that cardiometabolic side effects are common among patients initiating antipsychotic treatment. Additionally, multivariate regression analyses showed that pre-existing hypertension, type 2 diabetes mellitus, weight gain/obesity and cardiovascular disease/ events were significant predictors of developing dyslipidemia. These findings suggest that individuals with preexisting cardiometabolic conditions are at greater risk for dyslipidemia after initiating antipsychotic therapy for 
Table 4 Multivariate modeling

\begin{tabular}{|c|c|c|c|c|c|c|c|c|}
\hline \multirow[b]{3}{*}{ Predictor (baseline characteristics) } & \multicolumn{4}{|c|}{ Dyslipidemia During the 24-Month Follow-up Period } & \multicolumn{4}{|c|}{$\begin{array}{l}\text { Index Regimen with High Weight Gain Risk vs Low/ } \\
\text { No Risk }\end{array}$} \\
\hline & \multicolumn{2}{|l|}{ Commercial } & \multicolumn{2}{|l|}{ Medicaid } & \multicolumn{2}{|l|}{ Commercial } & \multicolumn{2}{|l|}{ Medicaid } \\
\hline & Odds Ratio & $P$-value & Odds Ratio & $P$-value & Odds Ratio & $P$-value & Odds Ratio & $P$-value \\
\hline Intercept & 0.02 & $<0.01$ & 0.03 & $<0.01$ & 3.52 & $<0.01$ & 2.92 & $<0.01$ \\
\hline Age & 1.05 & $<0.01$ & 1.04 & $<0.01$ & 0.99 & $<0.01$ & 0.99 & $<0.01$ \\
\hline Female versus male & 0.63 & $<0.01$ & 1.08 & 0.26 & 0.92 & 0.39 & 0.88 & $<0.01$ \\
\hline Race: non-white versus white ${ }^{a}$ & - & - & 0.84 & 0.03 & - & - & - & - \\
\hline Race: Black vs. White ${ }^{a}$ & - & - & - & - & - & - & 0.95 & 0.43 \\
\hline Race: Hispanic vs. White ${ }^{a}$ & - & - & - & - & - & - & 0.90 & 0.58 \\
\hline Race: Other/Unknown vs. White ${ }^{a}$ & - & - & - & - & - & - & 1.14 & 0.13 \\
\hline Charlson Comorbidity Index & - & - & - & - & 0.99 & 0.89 & 1.04 & 0.05 \\
\hline Weight gain/obesity & 1.07 & 0.81 & 1.43 & $<0.01$ & 0.75 & 0.11 & 0.88 & 0.09 \\
\hline Dyslipidemia & - & - & - & - & 1.01 & 0.94 & 0.92 & 0.28 \\
\hline Pre-diabetes & - & - & - & - & 1.16 & 0.61 & 1.07 & 0.64 \\
\hline Type 2 diabetes mellitus & 1.83 & $<0.01$ & 2.24 & $<0.01$ & 0.93 & 0.72 & 0.84 & 0.08 \\
\hline Hypertension & 1.19 & 0.27 & 1.46 & $<0.01$ & 1.06 & 0.67 & 1.00 & 0.99 \\
\hline Cardiovascular disease/events & 1.52 & 0.02 & 1.26 & 0.03 & 1.04 & 0.79 & 1.06 & 0.46 \\
\hline Cerebrovascular disease/events & - & - & - & - & 0.69 & 0.14 & 0.91 & 0.49 \\
\hline Obstructive sleep apnea & - & - & - & - & 1.57 & 0.06 & 0.77 & 0.07 \\
\hline Involuntary movements & - & - & - & - & 1.27 & 0.36 & 0.98 & 0.87 \\
\hline Seizures/convulsions & - & - & - & - & 1.04 & 0.86 & 0.86 & 0.20 \\
\hline Sedation/somnolence & - & - & - & - & 1.78 & 0.06 & 1.39 & 0.06 \\
\hline Suicide ideation or behavior & 1.36 & 0.23 & 1.01 & 0.95 & 1.24 & 0.22 & 1.00 & 0.98 \\
\hline Paranoid schizophrenia & 0.77 & 0.05 & 0.87 & 0.05 & 0.87 & 0.13 & 0.95 & 0.31 \\
\hline Disorganized schizophrenia & - & - & - & - & 1.23 & 0.49 & 0.97 & 0.81 \\
\hline Other schizophrenia & 0.80 & 0.11 & 0.81 & $<0.01$ & 0.78 & 0.01 & 1.03 & 0.54 \\
\hline Schizoaffective disorder & 0.91 & 0.65 & 0.96 & 0.67 & 0.97 & 0.81 & 0.99 & 0.83 \\
\hline Generalized anxiety disorder & - & - & - & - & 1.35 & 0.13 & 0.89 & 0.39 \\
\hline Other anxiety disorder & 1.20 & 0.31 & 1.14 & 0.18 & 1.13 & 0.31 & 1.12 & 0.13 \\
\hline Major depressive disorder & 1.10 & 0.58 & 1.38 & $<0.01$ & 0.82 & 0.09 & 1.22 & 0.01 \\
\hline Bipolar disorder & 0.74 & 0.29 & 1.31 & 0.06 & 1.32 & 0.13 & 1.06 & 0.55 \\
\hline Other mood disorder & 1.32 & 0.19 & 0.90 & 0.41 & 0.87 & 0.33 & 1.06 & 0.49 \\
\hline Conduct disorder & - & - & - & - & 1.37 & 0.18 & 1.04 & 0.76 \\
\hline Disruptive behavior disorder & - & - & - & - & 1.24 & 0.33 & 1.09 & 0.52 \\
\hline Drug abuse & 0.65 & 0.08 & 0.71 & $<0.01$ & 1.43 & $<0.01$ & 1.21 & $<0.01$ \\
\hline Alcohol abuse & 1.11 & 0.73 & 0.97 & 0.81 & 1.17 & 0.42 & 0.99 & 0.89 \\
\hline Other substance abuse/dependency & 0.75 & 0.20 & 0.91 & 0.22 & 0.97 & 0.81 & 0.95 & 0.35 \\
\hline Delusional disorders & - & - & - & - & 1.71 & $<0.01$ & 1.16 & 0.22 \\
\hline SSRI medication & - & - & - & - & 0.98 & 0.87 & 1.07 & 0.26 \\
\hline SNRI medication & - & - & - & - & 1.01 & 0.97 & 0.88 & 0.33 \\
\hline Anti-anxiety prescription & - & - & - & - & 0.91 & 0.39 & 0.93 & 0.26 \\
\hline Anticonvulsant medication & - & - & - & - & 0.98 & 0.86 & 1.20 & $<0.01$ \\
\hline Antihypertension medication & - & - & - & - & 0.97 & 0.83 & 0.95 & 0.48 \\
\hline Antidiabetic medication & - & - & - & - & 0.77 & 0.23 & 0.76 & 0.02 \\
\hline Lipid-lowering medication & - & - & - & - & 1.21 & 0.21 & 1.21 & 0.03 \\
\hline HWGR versus low or no risk & 1.13 & 0.40 & 0.89 & 0.10 & - & - & - & - \\
\hline MPR & 1.75 & $<0.01$ & 2.58 & $<0.01$ & - & - & - & - \\
\hline LAl antipsychotic in index regimen & 1.04 & 0.86 & 0.83 & 0.04 & - & - & - & - \\
\hline
\end{tabular}

a Race/ethnicity data available for Medicaid sample only

Abbreviations: HWGR High weight gain risk index treatment regimen, LAI Long-acting injectable, MPR Medication Possession Ratio, SNRI Serotonin and noradrenaline 
schizophrenia. While this finding associating underlying risk factors and the delveopment of dyslipidemia may be intuitive, the analyses futher investigated the role of the types and weight gain risk profiles of antipsychotic medication initiated at index date.

Index antipsychotic weight gain risk profile was not consistently associated with dyslipidemia or related cardiometabolic conditions at follow-up. This finding was somewhat unexpected, as weight gain and obesity, which are expected to be side effects of HWGR medications, have been robustly linked to dyslipidemia and other cardiometabolic conditions. However, it is notable that patients initiating HWGR antipsychotic regimens had lower rates of pre-index cardiometabolic comorbidities. Similar trends were observed in the commercially-insured sample. This finding suggests that pre-existing cardiometabolic conditions and treatments may be a factor influencing provider choice of antipsychotic medication. For example, patients already receiving antidiabetic medications were more likely to initiate on LWGR medications, and we hypothesize that this could be due to physicians channeling patients deemed to be high-risk for other cardiometabolic conditions towards LWGR medications to avoid additional weight gain. This is also consistent with evidence indicating that LWGR antipsychotic regimens are preferred to HWGR antipsychotic regimens in patients who have recently experienced weight gain [12]. In a single-site, proscpective study, Edlinger et al. found that patients' side effect profiles influenced the physician choice of antipshychotic more than patient demographics or other illness-related variables. Patients who experienced weight gain in the past were less likely to swich to olanzapine (HWGR medication), suggesting that the weight gain risk profile may have been considered in the prescribing decision.

Interestingly, and unexpectedly, these findings did not hold true when modeling predictors of a HWGR profile regimen. Although pre-existing cardiometabolic conditions had no significant impact on the odds of initiating on HWGR vs LWGR regimen among patients in either payer, Medicaid patients receiving anti-diabetic medications did have lower odds of initiating HWGR while those receiving lipid-lowering medication had higher odds. Fig. 1 depicts propensity scores suggesting some minor separation between patients who are predicted to receive HWGR vs LWGR regimens, but there did not appear to be clearly distinct groups. This finding suggests that while some patient channeling is expected to occur in the real-world setting, our analysis did not reveal a true separation by baseline patient characteristics that can completely explain why some patients initiate HWGR and some intiate LWGR.

In contrast to weight gain risk profile, adherence was an important factor contributing to risk for cardiometabolic conditions. Specifically, we found that medication adherence was significantly associated with developing dyslipidemia in both payer groups; this trend may occur because greater medication adherence leads to higher levels of exposure to antipsychotic medications, thereby increasing the risk for adverse side effects. It should be noted, however, that mediation adherence overall was poor, with around two-thirds of patients classified as not adhering to the index antipsychotic regimen. This finding is consistent with previous research [13, 14]. MPR is considered a valid indicator of medication adherence in

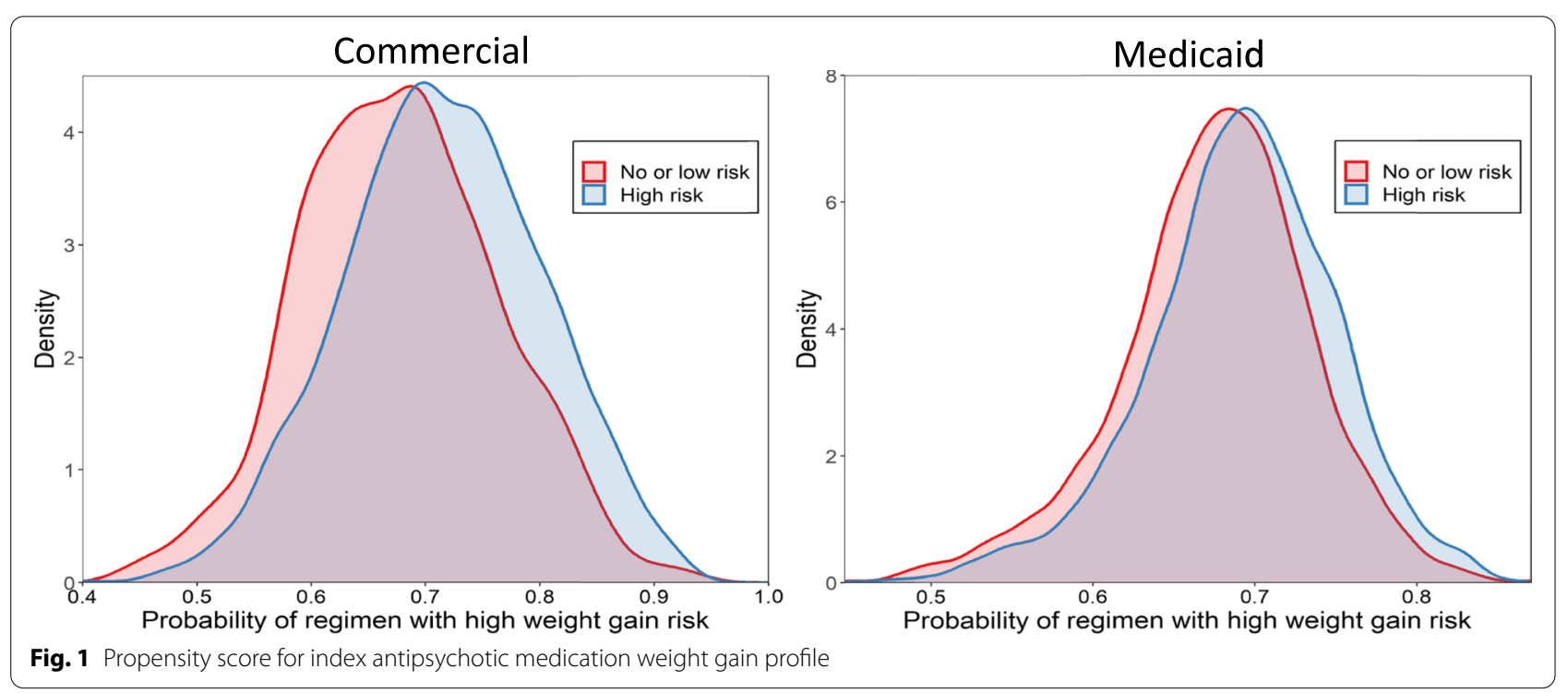


Table 4 (continued)

reuptake inhibitor, SSRI Selective serotonin reuptake inhibitor

claims data analyses, as it captures the total days supplied for filled pharmacy claims (i.e. pharmacy claims paid through insurance and not abandoned or not filled by the patient) [14]. The days supplied and relating this number to the total length of the follow-up period is an objective measure of the proportion of days in which the patient had the medication on hand. In this study, no difference was observed in how this adherence measure would perform in either the HWGR group or the LWGR group.

Claims-based studies have several important strengths, including longitudinal follow-up, lack of selection bias, and the availability of standardized data on health care services and filled prescriptions. Nonetheless, this study should be considered in the light of several limitations. First, claims are subject to incomplete, inaccurate, or missing data. In this study, all patients were continuously enrolled in medical and pharmacy benefits for the entire baseline and follow-up periods. Therefore, the data did not miss any claims that were submitted to the patients' insurance for reimbursement purpose. The analysis was conducted without data imputation considering the possibility of missing information was low. It should again be noted that schizophrenia patients tend to make little use of health care resources despite poor physical health [5]. This phenomena may lead to under-detection of underlying cardiometabolic comorbidities or risk factors, thus, results of this analysis may not be generalizable to the overall schizophrenia population.

A related concern is that pharmacy claims do not indicate whether the medication dispensed was taken as prescribed, potentially overestimating medication adherence. Additionally, the distinction of HWGR vs LWGR antipsychotic medications may be imperfect while research is still being conducted on the newer antipsychotics. Also, so-called "low weight gain" antipsychotics may actually lead to significant weight gain in certain populations. Physicians may be prescribing drugs based on many other considerations and not necessarily considering the weight gain profile of the specific antipsychotic medication.

Second, we cannot establish a causal relationship between antipsychotic initiation and cardiometabolic complications. Although we sought to avoid reciprocal causation by statistically adjusting for pre-index comorbidities, unobserved variables may account for the association between antipsychotic initiation and subsequent cardiometabolic complications, such as genetic predisposition, or unhealthy diet. Our analyses did not specifically investigate dosage-related impacts, where higher dosages may have great impacts on cardiovascular health. Similarly, claims data provide only limited information on factors that influence medication adherence. Patients who have greater insight into their illness and high levels of social support are more likely to adhere to treatment [15]. These factors may also predispose them to seek medical care, which may partly account for the association between medication adherence and being diagnosed with cardiometabolic complications.

\section{Conclusions}

Our findings indicate that there is unmet need for reducing cardiometabolic risk for patients initiating or continuing antipsychotic therapy. Cardiometabolic conditions often develop during the early stages of antipsychotic therapy, and the emergence of these conditions does not appear to be related to the weight gain risk profile of the index antipsychotic regimen. Inconsistencies in our findings across payers may point to unmeasured confounders, such as social determinants of health, influencing incidence of cardiometabolic conditions. While further research regarding these additional patient characteristics is certainly warranted, physicians and patients must also balance the potential cardiometabolic side effects of antipsychotic therapy against control of symptoms and risk for relapse. Finally, all patients with schizophrenia being treated with antipsychotic medications should undergo baseline screening and then regular monitoring of cardiac risk factors.

\section{Supplementary Information}

The online version contains supplementary material available at https://doi. org/10.1186/s12888-022-03746-0.

Additional file 1: Figure S1 Commercial and Medicaid Sample Selection.

Acknowledgements

Programming services were provided Helen Varker of IBM Watson Health. Statistical analysis services were provided Nicole Zimmerman of IBM Watson Health.

\section{Role of sponsor}

Merck participated in formulating the outline of the study but had no role in data selection. Although staff at Merck reviewed the manuscript, final approval for the decision to submit the manuscript was the sole decision of the authors.

\section{Previous presentations}

This research was presented in part at the 2021 American Psychiatric Association conference. 


\section{Authors' contributions}

All authors analyzed and interpreted data. ET and BL wrote the main manuscript. All authors read and approved the final manuscript.

\section{Funding}

This study was funded by Merck Sharp \& Dohme Corp., a subsidiary of Merck \& Co., Inc., Kenilworth, NJ, USA.

\section{Availability of data and materials}

The data that support the findings of this study are available from IBM Watson Health. IBM is the sole proprietor of the $\mathrm{IBM}^{\circledR}$ MarketScan Databases. This data analysis was conducted by IBM researchers under appropriate data use agreements. Restrictions apply to the availability of these data, which were used under license for this study. The datasets used and/or analysed during the current study are available on reasonable request by contacting Ellen Thiel, Researcher, IBM Watson Health—eriehle@us.ibm.com.

\section{Declarations}

\section{Ethics approval and consent to participate}

This research is not considered human subject research as it does not involve interactions with - or interventions among - participants, and only deidentified data was available. All database records are statistically de-identified and certified to be fully compliant with US patient confidentiality requirements set forth in the Health Insurance Portability and Accountability Act of 1996. Because this study used only de-identified patient records and did not involve the collection, use, or transmittal of individually identifiable data, this study was exempted from Institutional Review Board approval. Per The U.S. Department of Health and Human Services, Office of Human Research Protections regulations, the analysis of de-identified data does not constitute human subjects research as defined at 45 CRF 46.102 and as such it does not require IRB review [7].

\section{Consent for publication}

Not applicable.

\section{Competing interests}

RK and FC are employees of Merck Sharp \& Dohme Corp., a subsidiary of Merck \& Co., Inc., Kenilworth, NJ, USA who may own stock and/or hold stock options in Merck \& Co., Inc.,Kenilworth, NJ, USA. BL and ET are employed by IBM Watson Health which received funding from Merck to conduct this study.

\section{Author details}

${ }^{1}$ Merck \& Co., Inc., 2000 Galloping Hill Rd, Kenilworth, NJ 07033, USA. ${ }^{2}$ BBM

Watson Health, Ann Arbor, MI, USA.

Received: 11 June 2021 Accepted: 28 January 2022

Published online: 19 February 2022

\section{References}

1. GBD 2017 Disease and Injury Incidence and Prevalence Collaborators. Global, regional, and national incidence, prevalence, and years lived with disability for 354 diseases and injuries for 195 countries and territories, 1990-2017: a systematic analysis for the global burden of disease study 2017. Lancet. 2018;392(10159):1789-858.

2. American Psychiatric Association. Schizophrenia spectrum and other psychotic disorders. In: Diagnostic and Statistical Manual of Mental Disorders: DSM-5. 5th edn. American Psychiatric Publishing; 2013. p. 87-122.

3. Buckley PF, Foster A. Schizophrenia: current concepts and approaches to patient care. Am Health Drug Benefits. 2008;1(4):13-22.

4. Olfson M, Gerhard T, Huang C, Crystal S, Stroup TS. Premature mortality among adults with schizophrenia in the United States. JAMA Psychiat. 2015;72(12):1172-81.

5. Leucht S, Burkard T, Henderson J, Maj M, Sartorius N. Physical illness and schizophrenia: a review of the literature. Acta Psychiatr Scand. 2007;116(5):317-33.

6. Dayabandara M, Hanwella R, Ratnatunga S, Seneviratne S, Suraweera C, de Silva VA. Antipsychotic-associated weight gain: management strategies and impact on treatment adherence. Neuropsychiatr Dis Treat. 2017;13:2231-41.

7. Department of Health \& Human Services. Protection of Human Subjects. USA: CFR; 2000. p. 104-23.

8. Patel KR, Cherian J, Gohil K, Atkinson D. Schizophrenia: overview and treatment options. P T. 2014;39(9):638-45.

9. Campbell RH, Diduch M, Gardner KN, Thomas C. Review of cariprazine in management of psychiatric illness. Ment Health Clin. 2017;7(5):221-9.

10. Muench J, Hamer AM. Adverse effects of antipsychotic medications. Am Fam Physician. 2010;81(5):617-22.

11. Weiss C, Weiller E, Baker RA, et al. The effects of brexpiprazole and aripiprazole on body weight as monotherapy in patients with schizophrenia and as adjunctive treatment in patients with major depressive disorder: An analysis of short-term and long-term studies. Int Clin Psychopharmacol. 2018;33(5):255-60.

12. Edlinger $M$, Hofer $A$, Rettenbacher $M A$, et al. Factors influencing the choice of new generation antipsychotic medication in the treatment of patients with schizophrenia. Schizophr Res. 2009;113(2-3):246-51.

13. World Health Organization. The magnitude of the problemof poor adherence. In: Adherence to Long-Term Therapies: Evidence for Action. World Health Organization; 2003. p. 7-9.

14. Lacro JP, Dunn LB, Dolder CR, Leckband SG, Jeste DV. Prevalence of and risk factors for medication nonadherence in patients with schizophrenia: a comprehensive review of recent literature. J Clin Psychiatry. 2002;63(10):892-909.

15. Haddad P, Brain C, Scott J. Nonadherence with antipsychotic medication in schizophrenia: challenges and management strategies. Patient Relat Outcome Meas. 2014;5:43-62.

\section{Publisher's Note}

Springer Nature remains neutral with regard to jurisdictional claims in published maps and institutional affiliations.
Ready to submit your research? Choose BMC and benefit from:

- fast, convenient online submission

- thorough peer review by experienced researchers in your field

- rapid publication on acceptance

- support for research data, including large and complex data types

- gold Open Access which fosters wider collaboration and increased citations

- maximum visibility for your research: over 100M website views per year

At BMC, research is always in progress.

Learn more biomedcentral.com/submissions 